\title{
Mechanosynthesis and mechanochemical treatment of bismuthdoped vanadium phosphorus oxide catalysts for the partial oxidation of $n$-butane to maleic anhydride
}

\begin{abstract}
Three Bi-doped vanadyl pyrophosphate catalysts were prepared via dihydrate route (VPD method), which consisted of different preparation methods including mechanosynthesis, mechanochemical treatment, and the conventional reflux method. The catalysts produced by the above three methods were characterized by x-ray diffraction (XRD), scanning electron microscopy (SEM), and temperature programmed reduction (TPR). Catalytic evaluation for the partial oxidation of n-butane to maleic anhydride (MA) was also carried out. The XRD patterns of all the Bi-doped catalysts showed the main peaks of pyrophosphate phase. Lower intensity peaks were observed for the mechanochemically treated Bi-doped catalyst (VPDBiMill) with two additional small peaks corresponding to the presence of a small amount of V5+ phase. The TPR profiles showed that the highest amount of active oxygen species, i.e, V4+-O- pair, responsible for n-butane activation, was removed from VPDBiMill. Furthermore, from the catalytic test results, the graph of selectivity to MA as a function of the conversion of n-butane demonstrated that VPDBiMill was the most selective catalyst. This suggests that the mechanochemical treatment of vanadium phosphate catalyst (VPDBiMill) is a potential method to improve the catalytic properties for the partial oxidation of n-butane to maleic anhydride.
\end{abstract}

Keyword: mechanosynthesis, mechanochemical treatment, bismuth, vanadyl pyrophosphate 